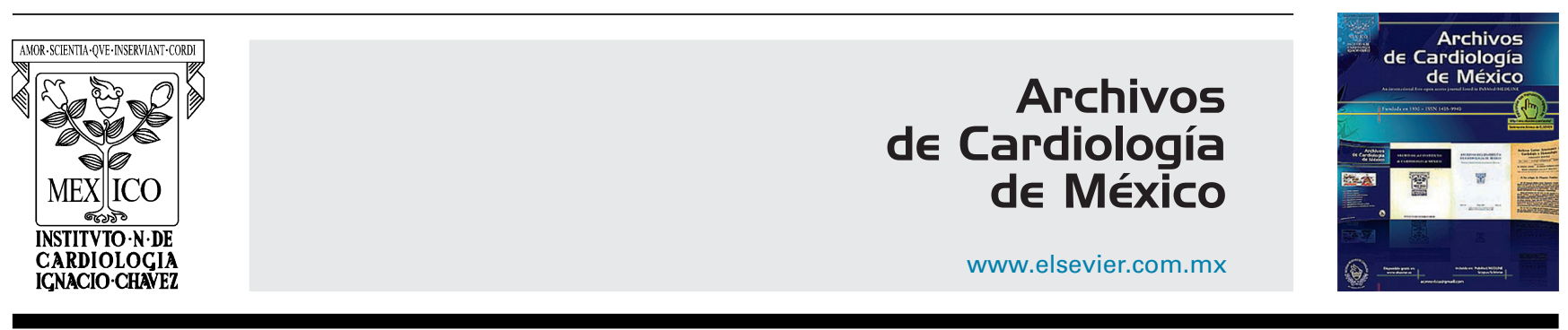

INVESTIGACIÓN CLÍNICA

\title{
Morbilidad posquirúrgica en pacientes pediátricos operados por cardiopatías congénitas en la UMAE de Yucatán
}

\section{Addy Castillo-Espínola*, Ana Velázquez-Ibarra, Aurea Zetina-Solórzano, Patricia Bolado-García y Gonzalo Gamboa-López}

Unidad Médica de Alta Especialidad, Servicio de Pediatría (Cardiología Pediátrica), División de Pediatría, Hospital de Especialidades CMN «lgnacio García Téllez», Mérida, Yucatán, México

Recibido el 27 de abril de 2016; aceptado el 26 de octubre de 2016

\author{
PALABRAS CLAVE \\ Cardiopatías \\ congénitas; \\ Periodo \\ posoperatorio; \\ Sepsis; \\ Síndrome de bajo \\ gasto; \\ Arritmias cardíacas; \\ México
}

\begin{abstract}
Resumen
Objetivo: Describir la evolución clínica de pacientes pediátricos operados por cardiopatías congénitas en la UMAE de Yucatán.

Métodos: Encuesta descriptiva sobre expedientes de pacientes pediátricos intervenidos quirúrgicamente por cardiopatía congénita del 1 de noviembre de 2011 al 30 de noviembre de 2013.

Resultados: Las cardiopatías más frecuentes fueron la persistencia del conducto arterioso (37.6\%) y la transposición de grandes vasos. La estancia en cuidados intensivos fue de 3 días (mediana). El $11.76 \%$ fallecieron por choque séptico (44.4\%) en la mayoría de los casos. Las complicaciones más frecuentes fueron sepsis $(5.9 \%)$, síndrome de bajo gasto $(4.7 \%)$, paro cardíaco, bloqueo $\mathrm{AV}$ y taquicardia ventricular ( $2.4 \%$ cada uno). Existe una correlación positiva moderada entre las complicaciones trans y posquirúrgicas y la sobrevida o muerte del paciente.

Conclusiones: El volumen de pacientes quirúrgicos es menor comparado con centros de referencia de cirugía cardiovascular. Existe una marcada tendencia a la realización de cirugías correctivas y paliativas en patologías específicas en pacientes con riesgos agregados o con «mala» anatomía cardiaca que impiden la corrección total de primera intención. Se deben realizar estudios epidemiológicos y clínicos prospectivos para conocer el comportamiento de las cardiopatías congénitas atendidas en la región.

( 2016 Instituto Nacional de Cardiología Ignacio Chávez. Publicado por Masson Doyma México S.A. Este es un artículo Open Access bajo la licencia CC BY-NC-ND (http://creativecommons. org/licenses/by-nc-nd/4.0/).
\end{abstract}

\footnotetext{
* Autor para correspondencia. Calle 34 \# 439 x 41, col. Industrial, Ex-terrenos «El Fénix», Mérida, Yucatán, México. CP 97150. Teléfono: +999 9225656 ext. 61677; teléfono celular: +999 1296313.

Correos electrónicos: addycastillo2@gmail.com, investigacion.umae.imss@gmail.com (A. Castillo-Espínola).
} 


\section{KEYWORDS}

Congenital heart

disease;

Post-operative

period;

Sepsis;

Low cardiac output;

Cardiac arrhythmias;

Mexico
Post-surgical morbidity in paediatric patients undergoing surgery for congenital heart disease in the UMAE of Yucatan, Mexico

\begin{abstract}
Objective: To describe the clinical course of paediatric patients undergoing surgery for congenital heart disease in UMAE of Yucatan.

Methods: Descriptive review was performed on the records of paediatric patients undergoing surgery for congenital heart disease from 1 November 2011 to 30 November 2013.

Results: The most frequent heart diseases were persistent ductus arteriosus (37.6\%) and transposition of the great vessels. The median intensive care stay was 3 days. Mortality was $11.76 \%$, with septic shock $(44.4 \%)$ in most cases. The most frequent complications were sepsis $(5.9 \%)$, low cardiac output syndrome $(4.7 \%)$, cardiac arrest, and AV block and ventricular tachycardia ( $2.4 \%$ each). There was a moderate positive correlation between surgical complications and survival or death.

Conclusions: The number of surgical patients is lower compared to reference centres for cardiovascular surgery. There is a marked tendency to perform corrective and palliative surgeries in specific disease in patients with added risk or 'bad' cardiac anatomy that prevent full correction at the first attempt. Prospective epidemiological and clinical studies should be conducted to understand the behaviour of congenital heart diseases treated in the region.

(C) 2016 Instituto Nacional de Cardiología Ignacio Chávez. Published by Masson Doyma México S.A. This is an open access article under the CC BY-NC-ND license (http://creativecommons. org/licenses/by-nc-nd/4.0/).
\end{abstract}

\section{Introducción}

Las cardiopatías congénitas son defectos estructurales del corazón y de los grandes vasos producidos durante el desarrollo embrionario y fetal ${ }^{1}$. Estas lesiones anatómicas pueden afectar a una o a varias de las 4 cámaras cardiacas, a los tabiques que las separan o a las válvulas o tractos de salida.

La etiología todavía no es clara. En muchas de las cardiopatías se consideran, hasta el momento, 3 principales causas: genética, ambiental y multifactorial ${ }^{2}$.

Su incidencia varía entre 3 a 8 por cada mil nacidos vivos ${ }^{3}$, y se estima que aproximadamente un $25 \%$ presentará, según el grado de complejidad, alteraciones en la dinámica de la circulación sanguínea que requieran intervención médica temprana, generalmente de tipo quirúrgico.

Las cardiopatías congénitas son las malformaciones más frecuentes. Su frecuencia en los países desarrollados se estima entre el 5.2 y el $12.5 \%$ de los recién nacidos vivos y alrededor del $1 \%$ en la población en general. En Estados Unidos de América las cardiopatías congénitas constituyen la causa principal de muerte relacionada con defectos neonatales, ocasionando más de 6,000 muertes por año. En nuestro país, el Instituto Nacional de Estadística, Geografía e Informática (INEGI) mencionó, en su reporte de 2011, un total de 29,050 defunciones en menores de un año, de las cuales 3,485 fueron por malformaciones congénitas del sistema circulatorio ${ }^{4}$. En 2013, en el estado de México se reportó que la incidencia general encontrada en 2 hospitales fue de 7.4/1,000 nacidos vivos. En los recién nacidos prematuros la incidencia fue de 35.6/1,000 y la de los recién nacidos a término fue de $3.68 / 1,000$. La cardiopatía más frecuente fue la persistencia del conducto arterioso en el grupo general. En los RN pretérmino predominó la combinación de la persistencia del conducto con la comunicación interauricular, y en los RN a término el defecto más frecuente fue la comunicación interauricular. La mortalidad específica asociada con los pacientes cardiópatas fue del $18.64 \%$, con un promedio de supervivencia de 437.92 días $^{5}$. Un estudio realizado en 2010 refirió que los defectos congénitos del corazón se ubican en el sexto lugar en menores de un año como causa de muerte infantil y como tercera causa de muerte en niños entre uno y 4 años $^{6}$. En México, el $85 \%$ de los defectos cardiacos son multifactoriales; el $50 \%$ de ellos requieren tratamiento quirúrgico, y de estos, el $30 \%$ se realizan con circulación extracorpórea ${ }^{7}$ (CEC).

Un análisis realizado en 2,257 pacientes con cardiopatías congénitas en el Hospital de Cardiología del Centro Médico Nacional Siglo XXI mostró que la persistencia del conducto arterioso representó el $20 \%$ de los casos, seguida de la comunicación interauricular con el $16.8 \%$, la comunicación interventricular con el 11\%, la tetralogía de Fallot y la atresia pulmonar con comunicación interventricular con el $9.3 \%$, la coartación aórtica y la estenosis pulmonar con el $3.6 \%$ y la conexión anómala de venas pulmonares con el $3 \%{ }^{6}$.

De acuerdo con su historia natural, las cardiopatías congénitas con manifestación clínica en el periodo neonatal tienen una mortalidad elevada, ya sea por tratarse de cardiopatías complejas o por la severidad en su presentación ${ }^{8}$. Es fundamental la sospecha de su presencia para iniciar, lo más pronto posible, la evaluación y el tratamiento médico, constituyendo su diagnóstico el paso intermedio para su necesaria corrección ${ }^{9}$. La Sociedad de Cirujanos Torácicos de Estados Unidos refirió que en 16 centros de ese país la mortalidad promedio fue entre el 3.7 y el $5.6 \%$ para el año 2001, y del 2.7 al 7.4\% para el año $2002^{10}$. Debido a los avances logrados en el manejo peri y postoperatorio, existe un incremento de cirugías correctivas con relación a los procedimientos 
paliativos (87.8\% contra $9.8 \%$, respectivamente $)^{11}$, lo que ha permitido realizar la corrección de los defectos en pacientes de menor edad y peso, reservando las cirugías paliativas solo para casos puntuales. Actualmente se realizan cirugías altamente complejas de manera rutinaria en recién nacidos pequeños, incluso prematuros con peso $\leq 2 \mathrm{~kg}$ y con edad gestacional de 32 semanas $^{12}$. Carísimo et al. ${ }^{1}$ reportaron en 2009 una serie de 91 pacientes con edades entre los 2 días y los 18 años con una sobrevida del $98 \%$ después de cirugías con CEC. Norwood et al. ${ }^{13}$ estudiaron los registros hospitalarios de Estados Unidos sobre la corrección de la transposición de grandes arterias con la cirugía de Jatene y encontraron que, en centros con menos de 10 operaciones al año, la mortalidad fue del $55 \%$, y en los hospitales con más de 50 cirugías anuales el promedio fue del $9 \%$. Checchia et al. ${ }^{14}$ realizaron en 2005 un estudio en 29 hospitales con 801 procedimientos de Norwood por 87 diferentes cirujanos y observaron que en donde se realizaba una o más cirugías de estas por mes la sobrevida fue del $78 \%$, en comparación con hospitales donde realizaron un procedimiento cada 2 meses o más y en donde la sobrevida fue del $59 \%$.

El tratamiento de las cardiopatías congénitas generalmente es quirúrgico ${ }^{3,15}$. El desarrollo tecnológico de sistemas complejos para la monitorización y derivación cardiopulmonar ha permitido el surgimiento de nuevos conceptos, como la cirugía toracoscópica, la cirugía robótica y la cirugía híbrida, así como la cirugía fetal ${ }^{16}$, para disminuir los riesgos transquirúrgicos y mejorar la sobrevida de estos pacientes. El perfeccionamiento de técnicas diagnósticas, quirúrgicas, anestésicas y de perfusión y el advenimiento de nuevos fármacos cardiológicos han mejorado la sobrevida de pacientes pediátricos con cardiopatías congénitas; sin embargo, no contamos con una base de datos a nivel nacional sobre la evolución posquirúrgica intrahospitalaria de estos pacientes.

Derivado de la falta de información estadística que refleje la realidad de nuestro país y considerando la tasa de natalidad anual en México, podemos estimar que cada año nacen alrededor de 20,000 niños con algún tipo de cardiopatía congénita. Lo reportado hasta el momento en el estado de Yucatán es que dichos padecimientos ocupan el lugar 13 a nivel nacional como causa de mortalidad, con una tasa del $1.98^{6}$. Por todo lo anterior, la realización de este trabajo fue con la finalidad de determinar la frecuencia de los resultados de la corrección quirúrgica de las cardiopatías congénitas. Esto permite considerar la necesidad de realizar estudios de pronóstico y de respuesta al tratamiento quirúrgico para identificar factores pronósticos que permitan implementar acciones de mejora y elevar la sobrevida, estandarizando los protocolos de atención y seguimiento y contribuyendo a disminuir la mortalidad, principalmente en los menores de 4 años de edad.

En los últimos años, en la Unidad Médica de Alta Especialidad de Mérida (Yucatán) se ha incrementado la atención a pacientes con cardiopatías congénitas, por lo que es importante conocer la prevalencia de los padecimientos tratados y la distribución de las variables que los acompañan.

\section{Métodos}

Previa autorización del protocolo de investigación, con número de registro R-2014-3203-1, se realizó una encuesta

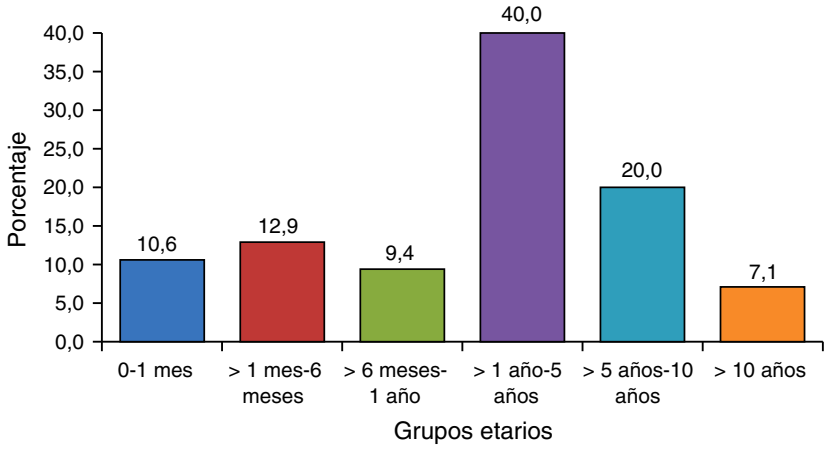

Figura 1 Proporción de los sujetos de estudio por grupos de edad.

de noviembre de 2011 a noviembre de 2013. Se incluyeron expedientes de 85 hombres y mujeres con cardiopatías congénitas intervenidos quirúrgicamente para corrección del defecto, con edades entre un día de vida a 14 años con 11 meses. No se incluyeron expedientes de pacientes que fueron referidos a otra unidad médica o a otra institución para su intervención quirúrgica. Se eliminaron los expedientes incompletos para la obtención de la información. Las variables consideradas fueron edad al momento de la intervención quirúrgica, sexo, tipo de cardiopatía diagnosticada, cirugía realizada, tiempo de CEC, tiempo de pinzamiento aórtico, complicaciones transquirúrgicas, días de estancia en unidad de cuidados intensivos, complicaciones posquirúrgicas, frecuencia de sobrevida al egreso de la unidad de cuidados intensivos y causa de la defunción. Se realizó análisis descriptivo calculando medias con desviaciones estándar de las variables numéricas y porcentaje para las variables nominales.

\section{Resultados}

De los 85 expedientes de pacientes intervenidos quirúrgicamente, el $50.6 \%$ fueron mujeres y el $49.4 \%$ fueron hombres, con edades comprendidas entre un día de vida y 14 años (fig. 1).

El 73\% fueron cardiopatías simples y el $27 \%$ restante fueron cardiopatías complejas. De las cardiopatías simples, las más frecuentes fueron persistencia del conducto arterioso (37.6\%), comunicación interventricular, comunicación interauricular (11.8\% cada una); de las cardiopatías complejas, la transposición de grandes vasos $(4.7 \%)$, la tetralogía de Fallot $(3.5 \%)$ y la atresia pulmonar $(2.4 \%)$ fueron las más frecuentes (figs. 2 y 3 , respectivamente).

El 61.2\% de los pacientes no presentaron comorbilidades. De estas, la más frecuente fue la desnutrición en cualquiera de sus grados $(9.5 \%)$, seguida del síndrome de Down en el $5.9 \%$ de los pacientes y de la HAP leve y moderada en el 4.7\% de los pacientes (fig. 4).

El $91.7 \%$ de las cirugías realizadas fueron correctivas y el $7 \%$ fueron paliativas. Un paciente requirió una cirugía de exploración cardiaca por atresia pulmonar con comunicación interventricular y comunicación interauricular; sin embargo, la anatomía presentada dificultó la cirugía correctiva y falleció en el posoperatorio inmediato debido a deterioro hemodinámico progresivo. El 31.8\% de las cardiopatías simples requirió CEC, al igual que el $18.8 \%$ de las cardiopatías 


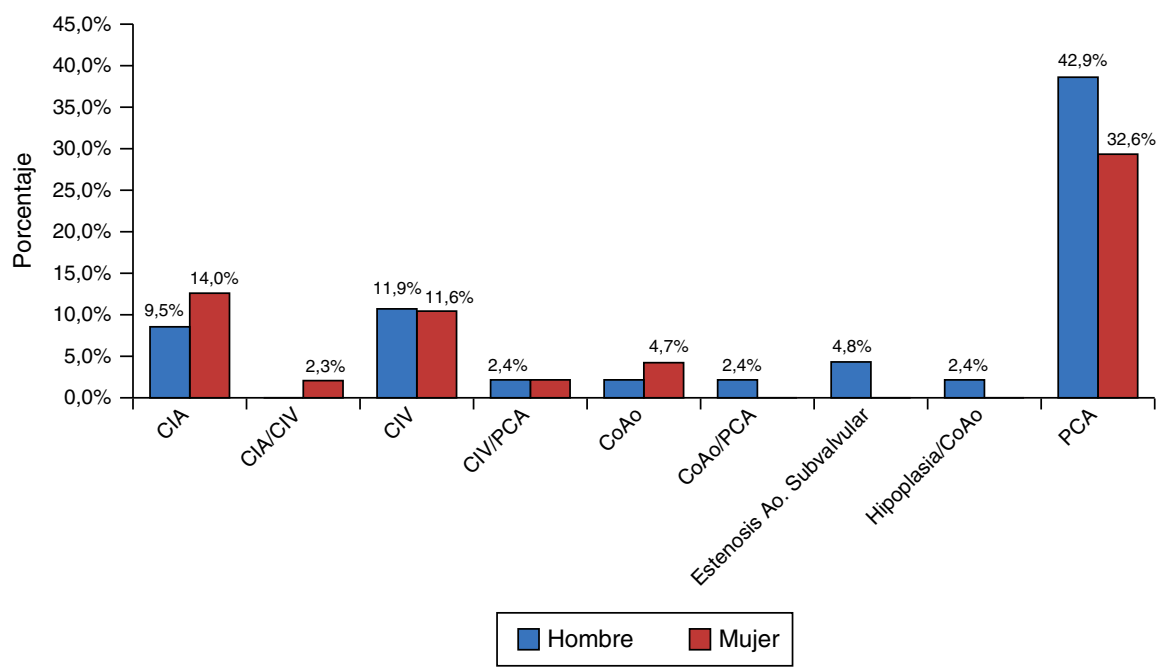

Figura 2 Cardiopatías simples. Ao: aórtica; CIA: comunicación interauricular; CIV: comunicación interventricular; CoAo: coartación aórtica; PCA: persistencia del conducto arterioso.

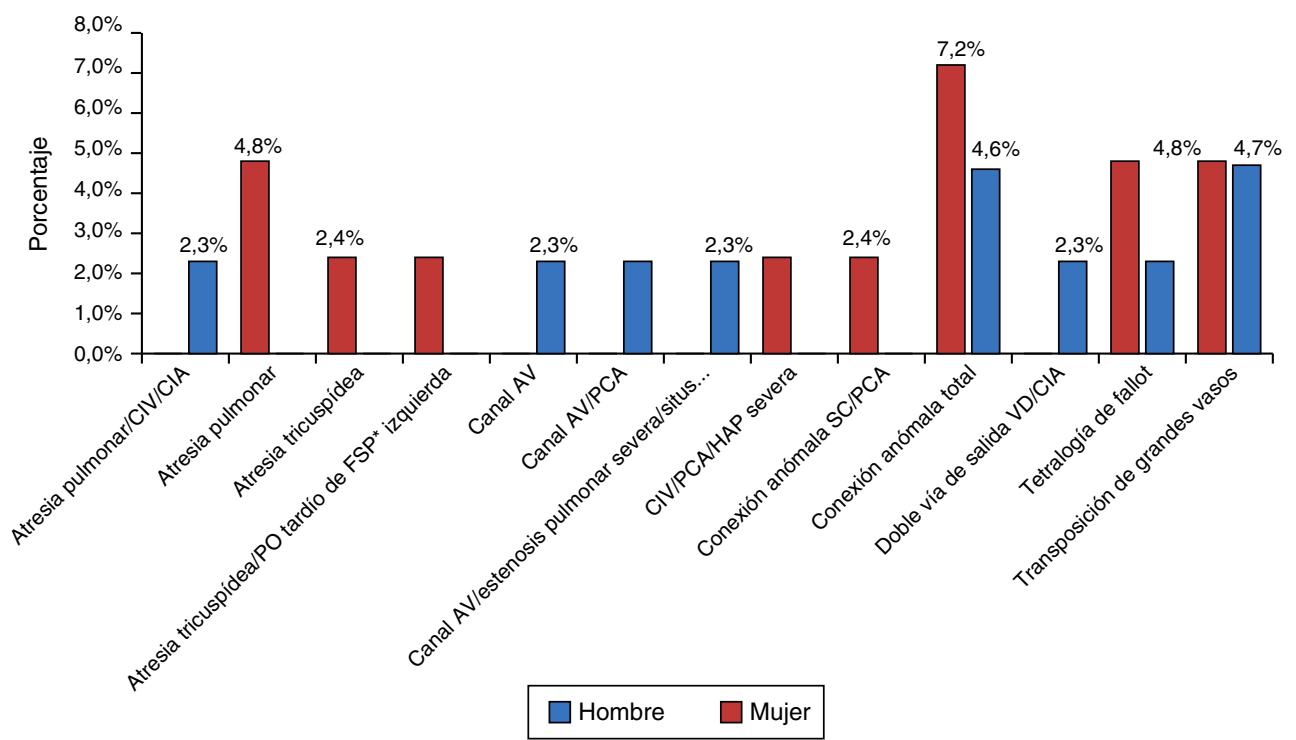

Figura 3 Cardiopatías complejas. Canal AV: canal auriculoventricular; CIA: comunicación interauricular; CIV: comunicación interventricular; FSP: fístula sistémico-pulmonar; PCA: persistencia del conducto arterioso; PO: postoperado.

Tabla 1 Tiempos de circulación extracorpórea y pinzamiento aórtico de las cardiopatías estudiadas

\begin{tabular}{lrl}
\hline & \multicolumn{1}{l}{ CEC } & PAo \\
\hline Cardiopatías simples & $50.77 \pm 18.51$ & $33.22 \pm 11.37$ \\
Cardiopatías complejas & $118.06 \pm 42.11$ & $91.12 \pm 36.25$ \\
\hline
\end{tabular}

CEC: circulación extracorpórea; Pao: pinzamiento aórtico.

Los valores se expresan en promedios $\pm \mathrm{DE}$.

complejas (fig. 5). El tiempo máximo de CEC y pinzamiento aórtico fue variable, dependiendo del tipo de procedimiento realizado (tabla 1 ).

La estancia promedio en las unidades de cuidados intensivos ( $\mathrm{UCl}$ ) tuvo una mediana de 3 días (fig. 6). Las complicaciones presentadas por los pacientes estudiados se dividieron en transquirúrgicas y posquirúrgicas (tabla 2). El $11.76 \%$ de los pacientes fallecieron. Todas las defunciones ocurrieron en el periodo posquirúrgico, y la causa más frecuente fue choque séptico (44.4\%), seguida de bloqueo $\mathrm{AV}$, síndrome de bajo gasto y choque cardiogénico $(22.2 \%$, cada una) y hemorragia pulmonar (11.1\%).

\section{Discusión}

Los últimos años han sido testigos de los avances en cirugía para la corrección de cardiopatías congénitas. Hay un incremento importante a nivel mundial en el número de recién nacidos sometidos a cirugías correctivas, realizándose hasta un $20 \%$ de las cirugías cardiacas durante el primer mes de vida. ${ }^{1}$ La edad de mayor predominio al momento de la cirugía fue entre uno y 5 años, y solo el $10.6 \%$ de los pacientes 


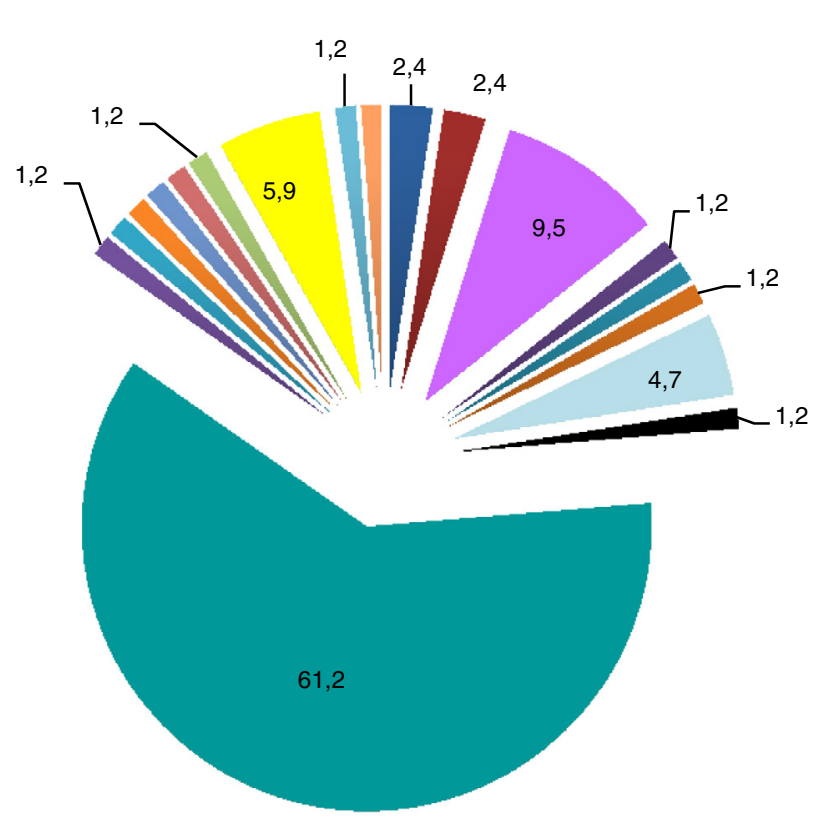

Asma
Trastornos de vesícula y vías biliares
Desnutrición
Epilepsia criptogénica
Epilesia/Asma
FSP
HAP leve/moderada
Mutismo aquinético secundario
Ninguna
Retraso psicomotor
Retraso psicomotor leve
RNPT 32 SDG/RCIU simétrico/DBP
RNPT 34 SDG/HAP severa/síndrome 35 SDG

Figura 4 Porcentaje de comorbilidades encontradas. DBP: displasia broncopulmonar; FSP: fístula sistémico-pulmonar; HAP: hipertensión pulmonar; RCIU: retardo en el crecimiento intrauterino; RNPT: recién nacido pretérmino; SDG: semanas de gestación.

se operaron en la etapa neonatal. Esto probablemente se debió a que nuestro hospital no es un centro de referencia para cirugía cardiovascular en congénitos y al envío tardío de los pacientes.

Carísimo et al. ${ }^{1}$ publicaron en 2009 un estudio sobre cardiopatías congénitas en 91 pacientes; la más frecuente fue la persistencia del conducto (24.2\%), seguida por la comunicación interventricular (14.3\%) y la comunicación interauricular (14.3\%). En nuestro país, un análisis de 2,257 pacientes realizado en el Hospital de Cardiología del Centro Médico Nacional Siglo XXI reportó que la persistencia del conducto arterioso se presentó en el $20 \%$ de los casos, la comunicación interauricular en el $16.8 \%$ y la comunicación interventricular en el $11 \%{ }^{6}$. Las frecuencias encontradas en nuestro estudio fueron similares. Sin embargo, el porcentaje de frecuencia de la persistencia del conducto arterioso es mayor a lo reportado probablemente por el número de sujetos estudiados y el tipo de patologías incluidas. De la misma manera, la frecuencia de las cirugías correctivas estuvo en relación con la frecuencia de las cardiopatías presentadas. Esto fue diferente a lo reportado en 2007 por Gallegos-Rivas et al. ${ }^{17}$ sobre 17,546 mujeres con hijos nacidos vivos en quienes se identificaron 98 defectos congénitos $(5.5 / 1,000$ recién nacidos vivos), de los cuales el $15.3 \%$ correspondió a cardiopatías congénitas en donde predominaron las cardiopatías complejas.

Las cardiopatías congénitas se acompañan de malformaciones en otros órganos y sistemas en por lo menos el $25 \%$

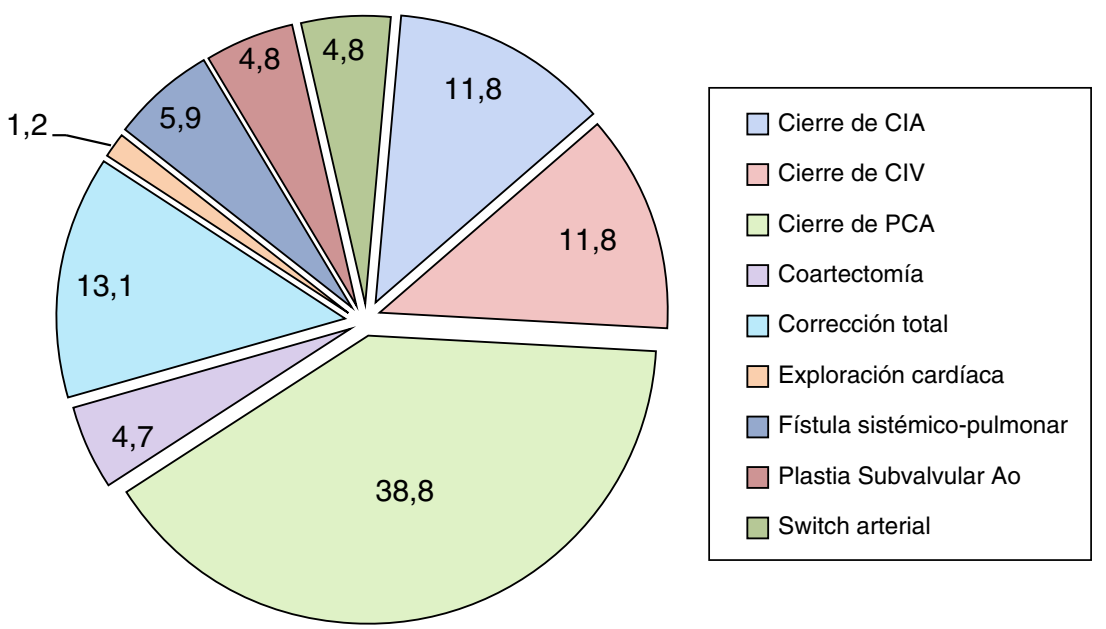

Figura 5 Porcentaje de cirugías realizadas. Ao: aórtica; CIA: comunicación interauricular; CIV: comunicación interventricular; PCA: persistencia del conducto arterioso. 


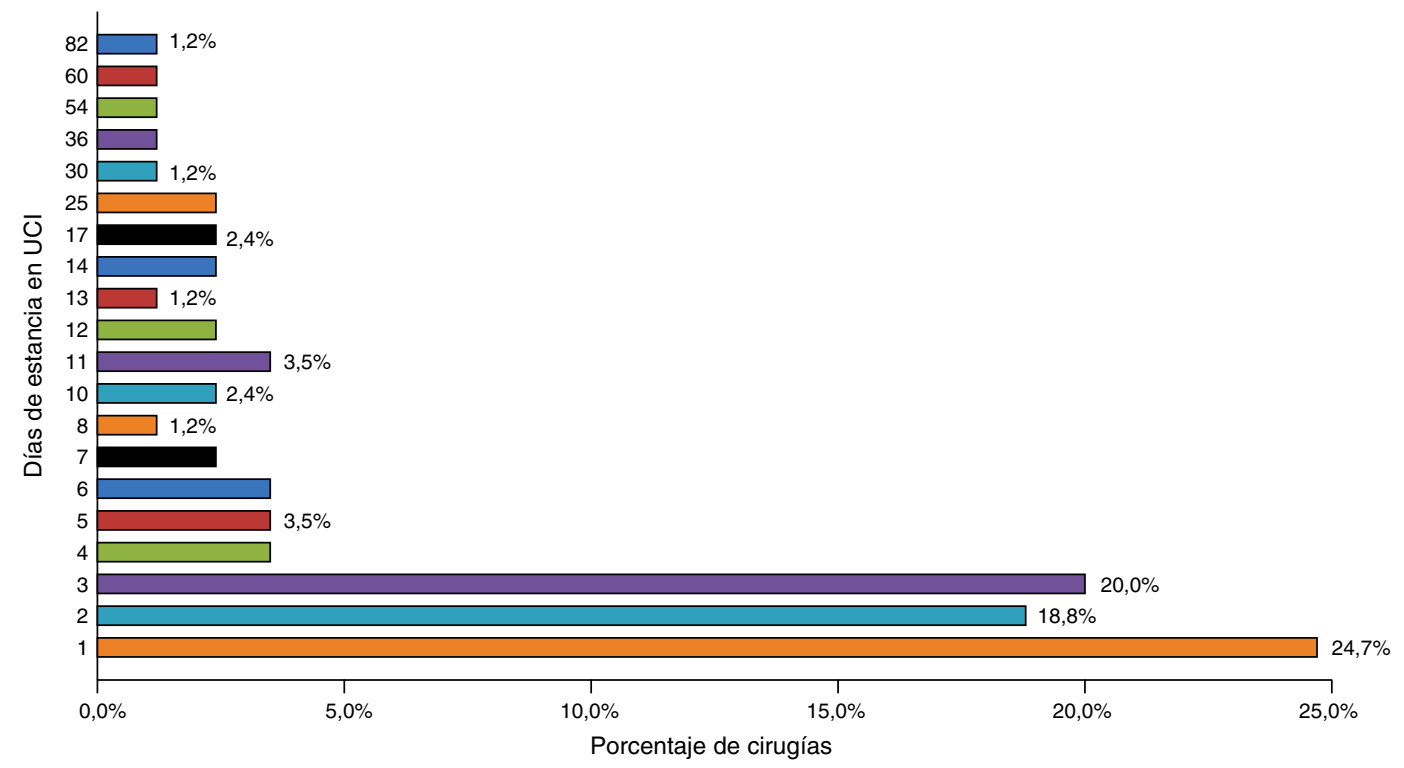

Figura 6 Proporción de cirugías por días de estancia en la unidad de cuidados intensivos (UCI).

Tabla 2 Complicaciones transquirúrgicas y posquirúrgicas de las cardiopatías estudiadas

\begin{tabular}{lr}
\hline Complicaciones transquirúrgicas & \\
Sin complicaciones & $91.8 \%$ \\
Acidosis & $1.2 \%$ \\
Bloqueo AV completo & $2.4 \%$ \\
Hipotensión & $1.2 \%$ \\
Mala anatomía & $1.2 \%$ \\
Paro cardíaco & $2.4 \%$ \\
Complicaciones posquirúrgicas & \\
Sin complicaciones & $68.2 \%$ \\
Bloqueo AV completo & $2.4 \%$ \\
Choque cardiogénico & $1.2 \%$ \\
Choque hipovolémico & $1.2 \%$ \\
Dehiscencia de herida quirúrgica & $1.2 \%$ \\
Enterocolitis necrosante 1A & $1.2 \%$ \\
Enfisema & $1.2 \%$ \\
Hipertensión & $1.2 \%$ \\
Lesión renal aguda & $1.2 \%$ \\
Mediastinitis & $1.2 \%$ \\
Neumonía nosocomial & $2.4 \%$ \\
Neumotórax & $1.2 \%$ \\
Paro cardíaco & $2.4 \%$ \\
Sepsis nosocomial & $7.1 \%$ \\
Síndrome de bajo gasto & $4.7 \%$ \\
Taquicardia ventricular & $2.4 \%$ \\
\hline AV: auriculoventricular. & \\
&
\end{tabular}

de los $\operatorname{casos}^{18}$. La anormalidad cromosómica más frecuente es el síndrome de Down, con una incidencia de 1/600 a 800 niños. En México se presenta en $1 / 420$ a 480 niños ${ }^{19}$. Weijerman et al. ${ }^{20}$ reportaron que la prevalencia de cardiopatías congénitas ocurrió en el 43\% de niños con trisomía 21 . Las malformaciones más frecuentemente asociadas a este tipo de pacientes incluyen el canal auriculoventricular, la persistencia del conducto arterioso, los defectos del tabique auricular y los del tabique ventricular.
En un estudio realizado en 2009, Mojica-Walteros et al. ${ }^{21}$ reportaron que el síndrome de Down se presentó en el $11 \%$ de los pacientes estudiados como una de las comorbilidades; sin embargo, la comorbilidad más frecuente fue la desnutrición (55.5\% de los pacientes). La HAP se presentó en el $4.5 \%$ de los pacientes estudiados como una complicación quirúrgica asociada con mortalidad. Datos similares encontramos en los sujetos estudiados. La desnutrición acompañó al 9.5\% de los pacientes, el síndrome de Down en el $5.9 \%$ y la HAP leve a moderada en el $4.7 \%$ de los casos.

Abdala et al. ${ }^{22}$ publicaron en 2008 un total de 213 cirugías, en donde el $64.8 \%$ requirieron CEC. El $50.6 \%$ de las cirugías realizadas en nuestro centro hospitalario requirieron CEC y, tomando en consideración las recomendaciones de la Academia Americana de Pediatría publicadas en 2002, no se cumplieron los criterios de eficacia de dichas guías, que estipulan que, para los centros que realizan cirugía cardiaca, estas deben ser anualmente un promedio de 100 cirugías cardiacas, de las cuales el $75 \%$ debieran requerir $\mathrm{CEC}^{23}$. Esto puede deberse a que nuestro hospital no es considerado como un centro de referencia para el manejo integral de las cardiopatías congénitas. Sin embargo, esta información coincide con una encuesta nacional realizada en 2009 por la Coordinación de los Institutos de la Secretaría de Salud ${ }^{24}$, donde se reportó que en Yucatán se realizaron 68 procedimientos quirúrgicos, de los cuales el 62\% requirió de CEC, acercándose a la media nacional reportada por el mismo estudio, que fue del $62.13 \%$. Aunque las cifras son mayores que las reportadas por nuestro estudio, no se cumple con los criterios que las guías proponen. Es probable que esto se deba a las diferencias entre las unidades médicas en las distintas regiones del país.

Las complicaciones secundarias a cirugías cardiacas abarcan una amplia variedad. Publicaciones recientes concluyen que las arritmias son comunes debido a factores de riesgo como el tiempo prolongado de CEC, duración del pinzamiento aórtico, corta edad, desnutrición, hipotermia profunda y parada circulatoria. Carisimo et al. ${ }^{1}$ reportaron en 2009 una frecuencia de arritmias del 5.5\%. Las 
complicaciones que encontramos fueron diferentes. Durante el periodo transquirúrgico, el bloqueo $\mathrm{AV}$ se presentó en el $2.4 \%$, al igual que el paro cardíaco. Entre el 10 y $20 \%$ de los pacientes postoperados de corazón desarrollan infecciones nosocomiales ${ }^{25}$, que pueden originarse en la herida quirúrgica, en los pulmones, en las vías urinarias, en las líneas invasivas o en el tracto gastrointestinal. Después de la cirugía cardiaca el choque séptico puede tener consecuencias devastadoras y conducir a falla multiorgánica, choque irreversible y muerte. Carísimo et al. ${ }^{1}$ encontraron una frecuencia del $4.4 \%$ del choque séptico en 91 pacientes, con una mortalidad del $50 \%$. De alguna manera, aunque en una proporción diferente, las complicaciones presentadas por los pacientes que estudiamos coincidieron con lo ya descrito. Una vez concluidas las intervenciones quirúrgicas, las complicaciones más frecuentes fueron sepsis, síndrome de bajo gasto, paro cardíaco, bloqueo AV y taquicardia ventricular. Además de las complicaciones infecciosas y hemodinámicas, se han reportado algunas de origen neurológico, gastrointestinal, renal, metabólico y nutricional. La incidencia de isquemia cerebral luego de cirugía cardiaca varía entre el 1 al 4\%, dependiendo del tipo de cirugía ${ }^{26}$; sin embargo, en nuestro estudio ningún paciente presentó esta complicación.

El resultado final de una intervención quirúrgica está dado por la relación entre la complejidad de la misma y el desempeño del equipo que está a cargo del paciente. En la actualidad, la supervivencia global de los recién nacidos con cardiopatía congénita se sitúa en alrededor del $85 \%{ }^{6}$. En 1995 Jenkins et al. ${ }^{27}$ realizaron un estudio con el objetivo de correlacionar el número de sujetos intervenidos y la mortalidad perioperatoria. Una muestra de 2,833 niños operados por cardiopatías congénitas en 37 centros hospitalarios permitió concluir que los que realizaban más de 300 cirugías al año tenían un menor porcentaje de mortalidad. Hannan et al. ${ }^{28}$ publicaron en 1998 una investigación observando que en los hospitales donde se llevaron a cabo menos de 100 cirugías pediátricas anuales de corazón tuvieron tasas de mortalidad mayor (8.26\%), en comparación con los centros donde se realizaron más de 100 cirugías por año, con una mortalidad del 5.95\%. Spiegelhalter ${ }^{29}$ reportó en 2002 que la mortalidad en hospitales de bajo volumen quirúrgico fue del $14.7 \%$, en comparación con el $10 \%$ de mortalidad en centros hospitalarios con mayor volumen quirúrgico. En nuestro estudio, la frecuencia de muerte tuvo similitud con lo reportado. Este último dato debe ser tomado con reserva, porque la muestra de sujetos estudiados fue pequeña; por esta razón, no realizó el cálculo de la tasa de morbilidad o mortalidad, dejando en claro la necesidad de realizar estudios prospectivos, epidemiológicos y clínicos que proporcionen una idea más clara y precisa de la historia natural y la evolución clínica de las cardiopatías congénitas. Una atención oportuna y adecuada incide en la disminución de la mortalidad en los pacientes con cardiopatías congénitas ${ }^{6}$.

\section{Conclusiones}

Aunque en nuestro centro hospitalario el volumen de pacientes quirúrgicos es menor a lo reportado por centros de referencia de cirugía cardiovascular, los resultados del tratamiento quirúrgico por cardiopatías congénitas hallados en este estudio son similares a los estudios publicados:
- El defecto congénito más frecuente es la persistencia del conducto arterioso y la complicación posquirúrgica que más se presentó fue el choque séptico.

- El grupo etario más afectado fue el de > 1-5 años.

- Más del 88\% lograron sobrevivir.

- La comorbilidad más frecuentemente asociada con los defectos congénitos del corazón fue la desnutrición, seguida del síndrome de Down y la HAP leve a moderada.

- Más del $63 \%$ de los pacientes estuvieron solo 3 días de estancia en la unidad de cuidados intensivos.

Este estudio representa el inicio de la planeación y el desarrollo de estrategias encaminadas a la racionalización de los recursos que permitan incrementar el volumen de pacientes quirúrgicos atendidos, beneficiando así a la población con la mejora de la calidad de la atención médica, aprovechando al máximo los recursos existentes para disminuir la mortalidad por cardiopatías congénitas. Actualmente realizamos cirugías correctivas de primera intención, dejando los procedimientos paliativos para patologías muy específicas en donde los riesgos agregados al paciente o la «mala» anatomía cardiaca no permiten la corrección total del defecto en una primera intención.

No existen estadísticas actuales sobre las cardiopatías congénitas. Las que están reportadas son subjetivas, debido a las variaciones regionales que existen en el país y el tipo de unidades de atención médica involucradas. Por lo tanto, existe una clara necesidad de realizar estudios prospectivos epidemiológicos y clínicos sobre las cardiopatías congénitas. Es por eso que consideramos que debe tomarse en cuenta la propuesta realizada por el Dr. Calderón Colmenero y su equipo de investigación sobre la regionalización de la atención de las cardiopatías congénitas:

Esta tiene como objetivo la racionalización de los recursos con énfasis en servicios médicos de alta especialidad con la finalidad de lograr un mejor resultado clínico para los pacientes. La evaluación debe ser constante a fin de poder realizar las adecuaciones pertinentes al programa. Los beneficios para la población se reflejarán en el número de casos atendidos, la mejora en la calidad de la atención, el óptimo aprovechamiento de los recursos existentes y la disminución de la mortalidad. Al no disponer de la frecuencia real de las cardiopatías congénitas en nuestro país, se ha tenido que considerar un promedio teórico, derivado de la información mundial disponible. Es importante conocer el número total de pacientes afectados con cardiopatías congénitas y saber la frecuencia de cada una de las variantes.

Las cardiopatías congénitas tienen diferencias regionales en su incidencia, por lo que es conveniente realizar estudios por regiones del país con la finalidad de determinar el comportamiento de la mismas en el contexto de su lugar de origen.

\section{Responsabilidades éticas}

Protección de personas y animales. Los autores declaran que para esta investigación no se han realizado experimentos en seres humanos ni en animales. 
Confidencialidad de los datos. Los autores declaran que han seguido los protocolos de su centro de trabajo sobre la publicación de datos de pacientes.

Derecho a la privacidad y consentimiento informado. Los autores declaran que en este artículo no aparecen datos de pacientes.

\section{Financiamiento}

Ninguno.

\section{Conflicto de intereses}

Los autores declaran no tener ningún conflicto de intereses.

\section{Bibliografía}

1. Carísimo M, Szwako R, Garay N. Cardiopatías congénitas, resultado del manejo perioperatorio en 18 meses. Experiencia en el Departamento de Cardiocirugía Centro Materno Infantil. UNA. Rev Chil Pediatr. 2011;82:358-9.

2. Perich RM. Cardiopatías congénitas más frecuentes. Pediatr Integral. 2008;12:807-18.

3. Bolio A, Ruiz GS, Romero P. Pronóstico de niños cardiópatas sometidos a cirugía de Fontan: experiencia de treinta años en el Hospital Infantil de México Federico Gómez. Bol Med Hosp Infant Mex. 2013;70:151-8.

4. INEGI: Estadística de mortalidad 2011.

5. Mendieta-Alcántara GG, Santiago-Alcántara E, Mendieta-Zerón $\mathrm{H}$, et al. Incidencia de las cardiopatías congénitas y los factores asociados a la letalidad en niños nacidos en dos hospitales del Estado de México. Gac Med Mex. 2013;149:617-23.

6. Calderón J, Cervantes JL, Curi PJ. Problemática de las cardiopatías congénitas en México. Propuesta de regionalización. Arch Cardiol Mex. 2010;80:133-40.

7. González-Vergara AB. Circulación extracorpórea en el paciente neonato con cardiopatía congénita. Rev Mexicana de Enfermería Cardiológica. 2004;12:69-75.

8. Klienman C, Seri I, Polin RA. Hemodynamics and Cardiology: Neonatology Questions and Controversies. 2. ${ }^{a}$ ed. Estados Unidos: Elsevier; 2012.

9. Kreutzer E, López R. Insuficiencia cardiaca. Cardiología Pediátrica. SCC. Mc Graw Hill; 2003. p. 223-4.

10. Jacobs JP, Mavroudis C, Jacobs ML, et al. Lessons learned from the data analysis of the second harvest (1998-2001) of the Society of Thoracic Surgeons Congenital Heart Surgery Database. Eur J Cardiothorac Surg. 2004;26:18-37.

11. Castañeda A. Congenital heart disease: A surgical historical perspective. Ann Thorac Surg. 2005;79:2217-20.

12. Andropoulos DB. En: Stayer SA, Russel IA, Mossad EB, editores. Recent Advances in Anesthesia for Congenital Heart Disease. 2. ${ }^{a}$ ed. USA: Johny Wiley \& Sons, Inc; 2011.
13. Norwood WI, Dobell AR, Freed MD, et al. Inmediate results of the arterial switch repair: A 20-Institution study. J Thorac Cardiovasc Surg. 1988;96:854-63.

14. Checchia PA, McCollegan J, Daher N, et al. The effect of surgical case volume on outcome after the Norwood procedure. J Thorac Cardiovasc Surg. 2005; 129:754-9.

15. Jacobs JP, Maruszewski B, Kurosawa $\mathrm{H}$, et al. Congenital heart surgery databases around the world: Do we need a global database? Semin Thorac Cardiovasc Surg Pediatr Card Surg Annu. 2010;13:3-19.

16. Riera-Kinkel C. Actualidades en el tratamiento quirúrgico de las cardiopatías congénitas. Rev Mex Pediatr. 2010;77: 214-23.

17. Gallegos-Rivas MC, Romero-Gutiérrez G, Pérez-López NM, et al. Defectos congénitos Mayres y múltiples en neonatos de mujeres atendidas en un hospital de tercer nivel. Ginecol Obstet Mex. 2007;75:247-52.

18. Buendía-Hernández A, Gloss G. Manejo de las cardiopatías congénitas: Estado del arte. Arch Cardiol Mex. 2003;73 Supl 1: 21-5.

19. Espínola-Zavaleta N, Soto ME, Romero-Gonzalez A, et al. Prevalence of congenital heart disease and pulmonary hypertension in Down's syndrome: An echocardiographic study. J Cardiovasc Ultrasound. 2015;23:72-7.

20. Weijerman ME, van Furth AM, van der Mooren MD, et al. Prevalence of congenital heart defects and persistent pulmonary hypertension of the neonate with Down syndrome. Eur J Pediatr. 2010;169:1195-9.

21. Mojica-Walteros CM, Guerra-Romero AR. Factores de riesgo para mortalidad quirúrgica en menores de un año con cardiopatía congénita. Bogotá: Universidad del Rosario, Fundación Cardioinfantil I.C.; 2009.

22. Abdala D, Lejbusiewicz G, Pose G, et al. Tratamiento quirúrgico de las cardiopatías congénitas: Resultados de 213 procedimientos consecutivos. Rev Chil Pediatr. 2008;79:90-7.

23. American Academy of Pediatrics. Guidelines for Pediatric Cardiovasular Center. Pediatrics. 2002;109:544-9.

24. Calderón-Colmenero J, de-la-Llata M, Vizcaíno A, et al. Atención médico-quirúrgica de las cardiopatías congénitas. Rev Invest Clin. 2011;63:344-52.

25. Levy I, Ovadia B, Erez E, et al. Nosocomial infections after cardiac surgery in infants and children: Incidence and risk factors. J Hosp Infect. 2003;53:111-6.

26. Ricotta JJ, Faggioli GL, Castilone A, et al. Risk factors for stroke after cardiac surgery: Buffalo Cardiac-Cerebral Study Group. J Vasc Surg. 1995;21:359-63.

27. Jenkins KJ, Newburger JW, Lock JE, et al. In-hospital mortality for surgical repair of congenital heart defects: Preliminary observations of variation by hospital case load. Pediatrics. 1995;95:323-30.

28. Hannan EL, Racz M, Kavey RE, et al. Pediatric cardiac surgery: The effect of hospital and surgeon volume on in-hospital mortality. Pediatrics. 1998;101:963-9.

29. Spiegelhalter DJ. Mortality and volume of cases in paediatric cardiac surgery: Retrospective study based on routinely collected data. BMJ. 2002;324:261-3. 